J. Korean Math. Soc. 49 (2012), No. 1, pp. 17-30

http://dx.doi.org/10.4134/JKMS.2012.49.1.017

\title{
ON BOUNDARY REGULARITY OF HOLOMORPHIC CORRESPONDENCES
}

\author{
NABIL OURimi
}

\begin{abstract}
Let $D$ be an arbitrary domain in $\mathbb{C}^{n}, n>1$, and $M \subset \partial D$ be an open piece of the boundary. Suppose that $M$ is connected and $\partial D$ is smooth real-analytic of finite type (in the sense of D'Angelo) in a neighborhood of $\bar{M}$. Let $f: D \rightarrow \mathbb{C}^{n}$ be a holomorphic correspondence such that the cluster set $c l_{f}(M)$ is contained in a smooth closed real-algebraic hypersurface $M^{\prime}$ in $\mathbb{C}^{n}$ of finite type. It is shown that if $f$ extends continuously to some open peace of $M$, then $f$ extends as a holomorphic correspondence across $M$. As an application, we prove that any proper holomorphic correspondence from a bounded domain $D$ in $\mathbb{C}^{n}$ with smooth real-analytic boundary onto a bounded domain $D^{\prime}$ in $\mathbb{C}^{n}$ with smooth real-algebraic boundary extends as a holomorphic correspondence to a neighborhood of $\bar{D}$.
\end{abstract}

\section{Introduction and main results}

The problem of analytic continuation of holomorphic maps (or correspondences) between real-analytic hypersurfaces is related to many fundamental questions in several complex variables, such as boundary regularity of proper holomorphic mappings (or correspondences), the regularity of CR maps and the classification of domains in complex spaces. This problem has attracted a lot of attention. For related results and without mentioning the entire list, we refer the reader to [15], [16], [25], [19], and [23]. In this paper we consider the problem of analytic continuation of holomorphic correspondences defined from a domain $D$ in $\mathbb{C}^{n}$ into $\mathbb{C}^{n}$ and sending in terms of cluster sets, a real-analytic hypersurface $M \subset \partial D$ into another real-algebraic hypersurface in $\mathbb{C}^{n}$ under the assumption that the correspondence extends continuously to some open peace of $M$. Our main theorem is the following:

Theorem 1. Let $D$ be an arbitrary domain in $\mathbb{C}^{n}, n>1$, and $M \subset \partial D$ be an open piece of the boundary. Suppose that $M$ is connected and $\partial D$ is smooth realanalytic of finite type in a neighborhood of $\bar{M}$. Let $f: D \rightarrow \mathbb{C}^{n}$ be a holomorphic

Received July 19, 2010

2010 Mathematics Subject Classification. 32H40, 32H35.

Key words and phrases. analytic sets, holomorphic correspondences, Segre varieties.

Supported by the King Saud University D. S. F. P. program. 
correspondence such that the cluster set $\mathrm{cl}_{f}(M)$ is contained in a smooth closed real-algebraic hypersurface $M^{\prime}$ in $\mathbb{C}^{n}$ of finite type. If $f$ extends continuously to some open peace of $M$, then it extends as a holomorphic correspondence across $M$.

By a smooth real-algebraic hypersurface we mean a real hypersurface in $\mathbb{C}^{n}$ globally defined by a polynomial equation $P(z, \bar{z})=0$. A similar result was proved in [1] for holomorphic mappings. Note that we do not require pseudoconvexity of $M$ or $M^{\prime}$. The proof of Theorem 1 is based on the idea of analytic continuation of holomorphic correspondences along Segre varieties and the construction of a family of ellipsoids used in [14]. Theorem 1 is already known if $M$ and $M^{\prime}$ are closed smooth real-analytic hypersurfaces of finite type and in additional $f$ is continuous on $M([23])$. For $n=2$ the result was proved in [24] in the real-analytic case under the additional condition that $c l_{f^{-1}}\left(M^{\prime}\right) \subset M$.

As an application of Theorem 1, one has the following:

Corollary 1. Let $f: D \rightarrow D^{\prime}$ be a proper holomorphic correspondence from a bounded domain in $\mathbb{C}^{n}$ with smooth real-analytic boundary into a bounded domain $\mathbb{C}^{n}, n>1$, with smooth real-algebraic boundary. Then $f$ extends as a holomorphic correspondence to a neighborhood of $\bar{D}$.

Proof. If $D$ is pseudoconvex which implies that $D^{\prime}$ is also pseudoconvex, then according to [23], $f$ extends as a holomorphic correspondence to a neighborhood of $\bar{D}$. If $D$ is not pseudoconvex, then $f$ extends as a holomorphic correspondence to a neighborhood of some boundary point of $D$ and the result follows from Theorem 1.

In [23], R. Shafikov and K. Verma used the result of [23], where the continuity of the correspondence was proved, to prove Corollary 1 in the case of proper holomorphic correspondences between bounded smooth real-analytic pseudoconvex domains. In the case of proper holomorphic mappings, the result was proved by R. Shafikov [20].

The structure of this article is as follows : In Section 2 we give basic definitions of holomorphic correspondences and Segre varieties. In Section 3 we study analytic sets extending the graph of holomorphic correspondences. In Section 4 we study analytic continuation of germs of holomorphic correspondences along Segre varieties. Finally, in Section 5 we present a proof of Theorem 1.

\section{Notations, definitions and preliminaries}

Let $D, D^{\prime}$ be domains in $\mathbb{C}^{n}$ and $A$ be a complex purely $n$-dimensional subvariety contained in $D \times D^{\prime}$. We denote by $\pi_{1}: A \rightarrow D$ and $\pi_{2}: A \rightarrow D^{\prime}$ the natural projections. When $\pi_{1}$ is proper, $\left(\pi_{2} \circ \pi_{1}^{-1}\right)(z)$ is a non-empty finite subset of $D^{\prime}$ for any $z \in D$ and one may therefore consider the multi-valued mapping $f=\pi_{2} \circ \pi_{1}^{-1}$. Such a map is called a holomorphic correspondence 
between $D$ and $D^{\prime} ; A$ is said to be the graph of $f$. Since $\pi_{1}$ is proper, in particular it is a branched analytic covering. Then there exist an $(n-1)$ dimensional complex-analytic subset $V_{f}$ of the graph of $f$ and an integer $m$ such that $\pi_{1}$ is an $m$-sheeted covering map from the set $A \backslash \pi_{1}^{-1}\left(\pi_{1}\left(V_{f}\right)\right)$ onto $D \backslash \pi_{1}\left(V_{f}\right)$. Hence, $f(z)=\left\{f^{1}(z), \ldots, f^{m}(z)\right\}$ for all $z \in D \backslash \pi_{1}\left(V_{f}\right)$ and the $f^{j}$ 's are distinct holomorphic functions in a neighborhood of $z \in D \backslash \pi_{1}\left(V_{f}\right)$. The integer $m$ is called the multiplicity of $f$ and $\pi_{1}\left(V_{f}\right)$ is its branch locus. One says that $f$ is irreducible if $A$ is irreducible as an analytic set and proper if both $\pi_{1}$ and $\pi_{2}$ are proper.

Given a holomorphic correspondence $f: D \rightarrow D^{\prime}$ with graph $\Gamma_{f} \subset D \times D^{\prime}$, one can find the system of canonical defining functions

$$
\phi_{I}(z, w)=\sum_{|J| \leq m} \phi_{I J}(z) w^{J},|I|=m,(z, w) \in \mathbb{C}^{n} \times \mathbb{C}^{n},
$$

where $\phi_{I J}(z) \in \mathcal{O}(D)$ and $\Gamma_{f}$ is precisely the set of common zeros of the functions $\phi_{I}(z, w)$ (see [6] for details). Let $z_{o} \in \partial D$ be a boundary point. We say that $f$ extends continuously at $z_{o}$ if and only if the holomorphic functions $\phi_{I J}$ extend continuously at $z_{o}$. We say that $\Gamma_{f}$ extends as an analytic set to $U \times \mathbb{C}^{n}$, where $U$ is a neighborhood of $z_{o}$, if there exists a closed complexanalytic set $\mathcal{A} \subset U \times \mathbb{C}^{n}$ of pure dimension $n$, which may possibly be reducible, such that, $\Gamma_{f} \cap\left\{(D \cap U) \times \mathbb{C}^{n}\right\} \subset \mathcal{A}$. If, in addition, the natural projection $\pi: \mathcal{A} \rightarrow U$ is proper, we say that $f$ extends as a holomorphic correspondence to a neighborhood $U$ of $z_{o}$.

The extension of $f$ as a holomorphic correspondence, at first, usually provided only a multi-valued extension which may be best interpreted as an analytic set extending the graph of $f$.

We will write $z=\left(z_{1},{ }^{\prime} z\right) \in \mathbb{C} \times \mathbb{C}^{n-1}$ for a point $z \in \mathbb{C}^{n}(n>1)$. Let $M$ be a smooth real-analytic hypersurface that contains the origin. By $\rho(z, \bar{z})$ we denote a real-analytic defining function of $M$ near 0 . In a small neighborhood $U$ of the origin, the complexification $\rho(z, \bar{w})$ of $\rho$ is well-defined by means of a convergent power series in $U \times U$. For $w \in U$, the associated Segre variety is defined as

$$
Q_{w}=\{z \in U: \rho(z, \bar{w})=0\} .
$$

By the implicit function theorem, it is possible to choose neighborhoods $U_{1} \subset \subset$ $U_{2}$ of the origin such that for any $w \in U_{1}, Q_{w}$ is a closed, complex hypersurface in $U_{2}$ and

$$
Q_{w}=\left\{\left(z_{1},{ }^{\prime} z\right) \in U_{2}: z_{1}=h\left({ }^{\prime} z, \bar{w}\right)\right\},
$$

where $h\left({ }^{\prime} z, \bar{w}\right)$ is holomorphic in ' $z$ and antiholomorphic in $w$. Following the terminology of [10], $U_{1}$ and $U_{2}$ are usually called a standard pair of neighborhoods of 0 . It can be shown that $Q_{w}$ is independent of the choice of the defining function. 
We denote by $S=S(U)$ the set of Segre varieties $\left\{Q_{w}, w \in U\right\}$ and $\lambda$ the so-called Segre map defined by

$$
\begin{aligned}
\lambda: \quad & U \rightarrow S \\
& w \mapsto Q_{w} .
\end{aligned}
$$

Let $I_{w}:=\left\{z \in U: Q_{w}=Q_{z}\right\}$ be the fiber of $\lambda$ over $Q_{w}$. For any $w \in M$, the set $I_{w}$ is a complex variety of $M$. We say that $M$ is essentially finite if for any $w \in M$ there exists a neighborhood $U_{w}$ of $w$ such that $I_{w} \cap U_{w}=\{w\}$. The set $S$ admits the structure of a complex-analytic variety of finite dimension such that the map $\lambda$ is a finite antiholomorphic branched covering. The set $I_{w}$ contains at most as many points as the sheet number of $\lambda$. We next list some important properties of $Q_{w}$ and $I_{w}$ (see e.g. [8] and [13]).

(a) $z \in Q_{w} \Longleftrightarrow w \in Q_{z}$.

(b) $z \in Q_{z} \Longleftrightarrow z \in M$.

(c) $I_{w}=\bigcap\left\{Q_{z}: z \in Q_{w}\right\}$.

(d) The Segre map $\lambda: w \mapsto Q_{w}$ is locally one-to one near strictly pseudoconvex points of $M$.

Let $f: D \rightarrow D^{\prime}$ be a holomorphic correspondence between domains in $\mathbb{C}^{n}$ with smooth real-analytic boundaries which extends as a holomorphic correspondence $F$ to a neighborhood of a point $p \in \partial D$. Assume that $p=0,0^{\prime} \in$ $f(0)$ and choose standard neighborhoods $U_{2} \supset \supset U_{1} \ni 0$ and $U_{2}^{\prime} \supset \supset U_{1}^{\prime} \ni 0^{\prime}$. Then we have the following invariance property for the Segre variety under $F$ (see [24]):

$$
\text { For all }\left(w, w^{\prime}\right) \in \operatorname{graph}(F) \cap\left(U_{1} \times U_{1}^{\prime}\right), \quad F\left(Q_{w}\right) \subset Q_{w^{\prime}}^{\prime}
$$

This means that any branch of $F$ maps any point from $Q_{w}$ to $Q_{w^{\prime}}^{\prime}$ for any point $w^{\prime} \in F(w)$.

Finally, recall that if $f: D \rightarrow \mathbb{C}^{n}$ is a holomorphic correspondence and $M \subset \partial D$, then the cluster set $c l_{f}(M)$ is defined as:

$$
\begin{aligned}
& c l_{f}(M)=\left\{w \in \mathbb{C}^{n} \cup\{\infty\}: \lim _{j \rightarrow \infty} \operatorname{dist}\left(z_{j}, M\right)=0\right. \text { and } \\
& \left.\qquad \lim _{j \rightarrow \infty} \operatorname{dist}\left(f\left(z_{j}\right), w\right)=0 \text { for some sequence }\left\{z_{j}\right\} \text { in } D\right\} .
\end{aligned}
$$

\section{Analytic sets extending the graph of holomorphic correspondences}

In this section, we study analytic sets extending the graph of holomorphic correspondences. More precisely we prove the following:

Theorem 2. Let $D$ be an arbitrary domain in $\mathbb{C}^{n}, n>1$, and $f: D \rightarrow$ $\mathbb{C}^{n}$ be a holomorphic correspondence. Let $M \subset \partial D$ be an open piece of the boundary. Suppose that $\partial D$ is smooth real-analytic and nondegenerate in an open neighborhood of $\bar{M}$ and the cluster set $c_{f}(M)$ is contained in a closed smooth real-algebraic nondegenerate hypersurface $M^{\prime} \subset \mathbb{C}^{n}$. If the graph of $f$ 
extends as an analytic set to $U \times \mathbb{C}^{n}$, where $U$ is a neighborhood of some point $p \in M$, then $f$ extends as a holomorphic correspondence to a neighborhood of $p$.

Recall that a hypersurface is called nondegenerate if it contains no complexanalytic set with positive dimension. Theorem 2 was proved for holomorphic mappings in the case of real-analytic hypersurfaces (see [9]). Related results were proved in [2] and in [17]. The methods of proof of all these results are based on the method of Segre varieties. The following example (appeared in [9]) shows that the assumption of the nondegeneracy of $M$ and $M^{\prime}$ can not be dropped.

Example. Let $D=\left\{\left(z_{1}, z_{2}\right) \in \mathbb{C}^{2}: \mathcal{R} e\left(z_{2}\right)+\left|z_{1}\right|^{2}<0\right\}$ and $D^{\prime}=\left\{\left(z_{1}^{\prime}, z_{2}^{\prime}\right) \in\right.$ $\left.\mathbb{C}^{2}: \mathcal{R e}\left(z_{2}^{\prime}\right)+\left|z_{1}^{\prime}\right|^{2}\left|z_{2}^{\prime}\right|^{2}<0\right\}$. The map $f:\left(z_{1}, z_{2}\right) \mapsto\left(z_{1} / z_{2}, z_{2}\right)$ is a biholomorphism from $D$ to $D^{\prime}$. The graph of $f$ is contained in $\left\{\left(z_{1}, z_{2}, z_{1}^{\prime}, z_{2}^{\prime}\right) \in\right.$ $\left.\mathbb{C}^{2} \times \mathbb{C}^{2}: z_{1}^{\prime} z_{2}^{\prime}-z_{1}=0, z_{2}^{\prime}=z_{2}\right\}$, then $f$ extends as an analytic set to a neighborhood of $\left(0,0^{\prime}\right)$. But $0^{\prime} \in c l_{f}(0)$ and $\infty \in c l_{f}(0)$. This shows that $f$ cannot be extended as a holomorphic correspondence to a neighborhood of 0 (since it is not continuous at 0 ). Note that in this example the boundary of $D^{\prime}$ is degenerate (it contains the complex line $z_{2}^{\prime}=0$ ).

\subsection{Proof of Theorem 2}

Without loss of generality, we may assume that $p=0$ and 0 is not in the envelope of holomorphy of $D$. Let $U$ be a neighborhood of 0 and $\mathcal{A} \subset U \times \mathbb{C}^{n}$ be the closed, complex-analytic set of dimension $n$ extending the graph $\Gamma_{f}$ of $f$. We may assume that $\mathcal{A}$ is irreducible, as otherwise consider only the irreducible component containing the graph of $f$. Let $\pi_{1}: \mathcal{A} \rightarrow U$ be the coordinate projection to the first component and let $E=\left\{z \in U: \operatorname{dim} \pi_{1}^{-1}(z) \geq 1\right\}$. We denote by $F: U \backslash E \rightarrow \mathbb{C}^{n}$ the multiple-valued map corresponding to $\mathcal{A}$; that is,

$$
F(w)=\left\{w^{\prime}:\left(w, w^{\prime}\right) \in \mathcal{A}\right\} .
$$

We denote by $S_{F}$ its branch locus (i.e., for $z \in U \backslash E, z \in S_{F}$ if the coordinate projection $\pi_{1}$ is not locally biholomorphic near $\pi_{1}^{-1}(z)$ ). The crucial point in the proof is to show that $\pi_{1}^{-1}(0) \cap E=\emptyset$ (i.e., $\pi_{1}^{-1}(0)$ is discrete). We denote $U^{-}=D \cap U$ and $U^{+}=U \backslash \bar{D}$. We begin by showing that $\mathcal{A}$ has points lying over $U^{+}$.

Lemma 1. $\mathcal{A} \cap\left(U^{+} \times \mathbb{C}^{n}\right) \neq \emptyset$.

Proof. We follow the ideas of [17]. By contradiction assume that $\mathcal{A} \cap\left(U^{+} \times\right.$ $\left.\mathbb{C}^{n}\right)=\emptyset$. It is clear that $\mathcal{A} \not \subset(M \cap U) \times \mathbb{C}^{n}$. Let $L$ be a complex line in $\mathbb{C}^{n}$ which contains 0 and is transverse to $M$ such that $\Gamma_{f} \cap\left\{\left(U^{-} \cap L\right) \times \mathbb{C}^{n}\right\} \neq \emptyset$. We may choose $U$ such that $U^{-} \cap L$ is connected. Let $\tilde{\mathcal{A}}$ be the irreducible component of $\mathcal{A} \cap\left\{(U \cap L) \times \mathbb{C}^{n}\right\}$ containing $\Gamma_{f} \cap\left\{\left(U^{-} \cap L\right) \times \mathbb{C}^{n}\right\}$ and $0^{\prime}$ be a point in $c l_{f}(0)$. The analytic set $\tilde{\mathcal{A}}$ has pure complex dimension 1 and it contains $\left(0,0^{\prime}\right)$. Moreover, $\tilde{\mathcal{A}} \not \subset(M \cap U) \times \mathbb{C}^{n}$. We consider two cases: 
- If $\tilde{\mathcal{A}} \cap\left\{(M \cap U) \times \mathbb{C}^{n}\right\}$ is discrete, then by the continuity principle we deduce that $\left(0,0^{\prime}\right)$ is in the envelope of holomorphy of $U^{-} \times \mathbb{C}^{n}$.

- If $\tilde{\mathcal{A}} \cap\left\{(M \cap U) \times \mathbb{C}^{n}\right\}$ is not discrete, then no open subset of $\tilde{\mathcal{A}}$ can be contained in $\tilde{\mathcal{A}} \cap\left\{(M \cap U) \times \mathbb{C}^{n}\right\}$. Now, the strong disc theorem shows that $\left(0,0^{\prime}\right)$ is again in the envelope of holomorphy of $U^{-} \times \mathbb{C}^{n}$.

Hence, 0 is in the envelope of holomorphy of $D$. Indeed, if $g \in \mathcal{O}\left(U^{-}\right)$, we may regard it as a function $\tilde{g} \in \mathcal{O}\left(U^{-} \times \mathbb{C}^{n}\right)$. Then $\tilde{g}$ extends to a neighborhood of $\left(0,0^{\prime}\right)$ and the uniqueness theorem shows that the extension of $\tilde{g}$ is also independent of the variables $z^{\prime} \in \mathbb{C}^{n}$. It follows that $g$ extends holomorphically across 0 . This is a contradiction; since 0 is not in the envelope of holomorphy of $D$.

As a consequence of Lemma 1, we deduce the following result.

Lemma 2. There exists an open set $\Gamma \subset M \cap U$ such that $f$ extends as a holomorphic correspondence to a neighborhood of $U^{-} \cup \Gamma$, and the graph of $f$ near any point $\left(z, z^{\prime}\right), z \in \Gamma$ and $z^{\prime} \in f(z)$, is contained in $\mathcal{A}$. Moreover, $0 \in \bar{\Gamma}$.

Proof. Let $V_{F}=\left\{\left(z, z^{\prime}\right) \in \mathcal{A}: z \in S_{F}\right\}$. Since the complex dimension of $V_{F}$ is at most $n-1$ (because $\mathcal{A}$ is irreducible and the projection $\pi_{1}$ is locally biholomorphic in an open set of $\mathcal{A}$ ), then $\mathcal{A} \backslash V_{F}$ is connected by paths. Without loss of generality we may assume that $M \cap U=\partial D \cap U$. Let $(a, b) \in \Gamma_{f} \cap$ $\left(\mathcal{A} \backslash V_{F}\right) \cap\left(U^{-} \times \mathbb{C}^{n}\right)$ and by Lemma 1 choose $\left(a^{\prime}, b^{\prime}\right) \in\left(\mathcal{A} \backslash V_{F}\right) \cap\left(U^{+} \times \mathbb{C}^{n}\right)$, and connect them by a path $\gamma \subset \mathcal{A} \backslash V_{F}$. It follows that $\pi_{1}(\gamma) \cap M \neq \emptyset$. Let $z_{o}$ be the point where $\pi_{1}(\gamma)$ first intersects $M$. Then $f$ extends as a holomorphic correspondence along $\pi_{1}(\gamma)$ from $a$ to $z_{o}$ and the graph of $f$ over this part of $\pi_{1}(\gamma)$ is contained in $\mathcal{A} \backslash V_{F}$. It follows that $z_{o}$ is a point of holomorphic extendability as a correspondence for $f$. The second part follows from the fact that $U$ may be chosen arbitrarily small.

The proof of Theorem 2 uses some ideas of R. Shafikov developed in [19] to study the analytic continuation of holomorphic correspondences and equivalence of domains. First, we need the following lemma whose proof is deferred until the end of this section.

Lemma 3. There exists a holomorphic change of variables such that in the new coordinates $Q_{0} \not \subset E$.

As a consequence, we deduce the following:

Lemma 4. $\pi_{1}^{-1}(0)$ is discrete.

Proof. It suffices to show that $0 \notin E$. In view of Lemma 3, we may assume that $Q_{0} \not \subset E$. By contradiction, suppose that $0 \in E$. It follows that there exist a point $b \in Q_{0}$ and a small neighborhood $U_{b} \ni b$ such that $U_{b} \cap E=\emptyset$. We may choose $U$ and $U_{b}$ so small such that for any $z \in U$, the set $Q_{z} \cap U_{b}$ is 
non-empty and connected. Let $\Sigma=\left\{z \in U: Q_{z} \cap U_{b} \subset S_{F}\right\}$. Following the ideas in [8] and [10], we define

$$
X=\left\{\left(w, w^{\prime}\right) \in(U \backslash \Sigma) \times \mathbb{C}^{n}: F\left(Q_{w} \cap U_{b}\right) \subset Q_{w^{\prime}}^{\prime}\right\} .
$$

We follow the convention of using the right prime to denote the objects in the target domain. For instance, $Q_{w^{\prime}}^{\prime}$ will mean the Segre variety of $w^{\prime}$ with the respect to the hypersurface $M^{\prime}$.

We prove the following properties of $X$.

Claim 1.

i) $X$ is not empty;

ii) $X$ is a complex-analytic set in $(U \backslash \Sigma) \times \mathbb{C}^{n}$;

iii) $X$ is closed in $(U \backslash \Sigma) \times \mathbb{C}^{n}$;

iv) $\Sigma \times \mathbb{C}^{n}$ is a removable singularity for $X$.

Proof. i) According to Lemma 2, there exists a sequence $\left\{a_{j}\right\} \subset \Gamma \backslash(E \cup \Sigma)$ such that $a_{j} \rightarrow 0$ as $j \rightarrow \infty$ and $f$ extends as a holomorphic correspondence in a neighborhood of each $a_{j}$. It follows from the invariance property of Segre varieties under holomorphic correspondences [24] that for every $a_{j}$ there exists a neighborhood $U_{j} \ni a_{j}$ such that $F\left(Q_{a_{j}} \cap U_{j}\right) \subset Q_{a_{j}^{\prime}}^{\prime}$ for all $a_{j}^{\prime} \in F\left(a_{j}\right)$. This implies that $F\left(Q_{a_{j}} \cap U_{b}\right) \subset Q_{a_{j}^{\prime}}^{\prime}$. Thus, $\left(a_{j}, a_{j}^{\prime}\right) \in \mathcal{A}$ and therefore $X \neq \emptyset$.

ii) Let $\left(w, w^{\prime}\right) \in X$. Consider an open simply connected set $\Omega \subset U_{b} \backslash S_{F}$ such that $Q_{w} \cap \Omega \neq \emptyset$. The branches of $F$ are globally defined in $\Omega$. Since $Q_{w} \cap U_{b}$ is connected, the inclusion $F\left(Q_{w} \cap U_{b}\right) \subset Q_{w^{\prime}}^{\prime}$ is equivalent to

$$
F^{j}\left(Q_{w} \cap \Omega\right) \subset Q_{w^{\prime}}^{\prime}, \quad j=1, \ldots, m,
$$

where the $F^{j}$ denote the branches of $F$ in $\Omega$. We denote by $P^{\prime}\left(w^{\prime}, \bar{w}^{\prime}\right)$ a defining polynomial function of $M^{\prime}$. The inclusion $F^{j}\left(Q_{w} \cap \Omega\right) \subset Q_{w^{\prime}}^{\prime}, j=1, \ldots, m$ can be expressed as

$$
P^{\prime}\left(F^{j}(z), \bar{w}^{\prime}\right)=0 \text { for any } z \in Q_{w} \cap \Omega, \quad j=1, \ldots, m .
$$

We can choose $\Omega$ in the form $\Omega=\Omega_{1} \times{ }^{\prime} \Omega \subset \mathbb{C} \times \mathbb{C}^{n-1}$ such that

$$
Q_{w}=\left\{\left(k\left({ }^{\prime} z, \bar{w}\right),{ }^{\prime} z\right),{ }^{\prime} z \in{ }^{\prime} \Omega\right\}
$$

and

$$
P^{\prime}\left(F^{j}\left(k\left({ }^{\prime} z, \bar{w}\right),{ }^{\prime} z\right), \bar{w}^{\prime}\right)=0 \quad \text { for any }{ }^{\prime} z \in{ }^{\prime} \Omega .
$$

Thus, $X$ is defined by an infinite system of holomorphic equations in $\left(\bar{w}, \bar{w}^{\prime}\right)$. By the Noetherian property of the ring of holomorphic functions, we can choose finitely many points ${ }^{\prime} z^{1}, \ldots,{ }^{\prime} z^{m}$ so that (3.1) can be written as a finite system

$$
\sum_{|J| \leq d^{\prime}} \alpha_{J}^{k}(w) w^{\prime J}=0
$$

where $k=1, \ldots, m, d^{\prime}$ is the degree of $P^{\prime}$ in $w^{\prime}$ and $\alpha_{J}^{k}$ are holomorphic functions in $w$. Thus, $X$ is a complex-analytic set in $(U \backslash \Sigma) \times \mathbb{C}^{n}$. 
iii) The set $X$ is closed in $(U \backslash \Sigma) \times \mathbb{C}^{n}$. Indeed; let $\left(w^{j}, w^{\prime j}\right)$ be a sequence in $X$ that converges to $\left(w_{o}, w_{o}^{\prime}\right) \in(U \backslash \Sigma) \times \mathbb{C}^{n}$ as $j \rightarrow \infty$. Since $Q_{w^{j}} \rightarrow Q_{w_{o}}$ and $Q_{w^{\prime j}}^{\prime} \rightarrow Q_{w_{o}^{\prime}}^{\prime}$, from the inclusion $F\left(Q_{w^{j}} \cap U_{b}\right) \subset Q_{w^{\prime j}}^{\prime}$ we obtain

$$
F\left(Q_{w_{o}} \cap U_{b}\right) \subset Q_{w_{o}^{\prime}}^{\prime}
$$

which implies that $\left(w_{o}, w_{o}^{\prime}\right) \in X$ and thus, $X$ is a closed set.

iv) Now, let us show that $\Sigma \times \mathbb{C}^{n}$ is a removable singularity for $X$. Let $t \in \Sigma$. It follows that $\bar{X} \cap\left(\{t\} \times \mathbb{C}^{n}\right) \subset\{t\} \times\left\{z^{\prime}: F\left(Q_{t} \cap U_{b}\right) \subset Q_{z^{\prime}}^{\prime}\right\}$. If $w^{\prime} \in F\left(Q_{t} \cap U_{b}\right) \subset Q_{z^{\prime}}^{\prime}$, then $z^{\prime} \in Q_{w^{\prime}}^{\prime}$. Since $\operatorname{dim}_{\mathbb{C}} Q_{w^{\prime}}^{\prime}=n-1$, then $\left\{z^{\prime}: F\left(Q_{t} \cap U_{b}\right) \subset Q_{z^{\prime}}^{\prime}\right\}$ has dimension at most $2 n-2$ and $\bar{X} \cap\left(\Sigma \times \mathbb{C}^{n}\right)$ has $2 n$-dimensional measure zero. Now, Bishop's theorem can be applied to conclude that $\Sigma \times \mathbb{C}^{n}$ is a removable singularity for $X$.

Now, we continue with the proof of Lemma 4. According to Lemma 2, there exists a sequence $\left\{a_{j}\right\} \subset \Gamma \backslash(E \cup \Sigma)$ such that $a_{j} \rightarrow 0$ as $j \rightarrow \infty$ and $f$ extends as a holomorphic correspondence in a neighborhood of each $a_{j}$. For small neighborhoods $U_{j} \ni a_{j}$ we have:

$$
\left.X\right|_{U_{j} \times \mathbb{C}^{n}}=\left.\mathcal{A}\right|_{U_{j} \times \mathbb{C}^{n}}
$$

We denote by $\bar{X}$ the closure of $X$ in $U \times \mathbb{C}^{n}$. Without loss of generality we may assume that $\bar{X}$ is irreducible. In view of (3.3) and by the uniqueness theorem (see for instance $[6]$ ) we deduce that $\bar{X}=\mathcal{A}$. Let $\hat{F}$ be the multiple-valued mapping corresponding to $\bar{X}$. By construction, for any $a^{\prime} \in \hat{F}(0), \hat{F}(0)=I_{a^{\prime}}^{\prime}$. Since $0^{\prime} \in \hat{F}(0) \cap M^{\prime}$, it follows that $\hat{F}(0) \subset M^{\prime}$. Therefore $\hat{F}(0)$ is discrete, since $M^{\prime}$ is nondegenerate. This contradicts the fact that $0 \in E$ and completes the proof of Lemma 4.

\subsection{Conclusion of the proof of Theorem 2}

In view of Lemma 4 , there exist neighborhoods $U_{0}$ of 0 and $U^{\prime}$ of $M^{\prime}$ such that $U_{0} \cap E=\emptyset$ and $\mathcal{A} \cap\left(\{0\} \times \partial U^{\prime}\right)=\emptyset$. We may shrink $U_{0}$ such that $\mathcal{A} \cap\left(U_{0} \times \partial U^{\prime}\right)=\emptyset$; as otherwise, there exists a sequence $\left(z_{j}, z_{j}^{\prime}\right)_{j}$ in $\mathcal{A}$ such that $\left(z_{j}\right)_{j}$ converges to 0 and $\left(z_{j}^{\prime}\right)_{j}$ converges to $z_{o}^{\prime} \in \partial U^{\prime}$. This implies that $\left(0, z_{o}^{\prime}\right) \in \mathcal{A}$ and $z_{o}^{\prime} \in \partial U^{\prime}$ : a contradiction. This shows that $\mathcal{A} \cap\left(U_{0} \times U^{\prime}\right)$ defines a holomorphic correspondence from $U_{0}$ to $U^{\prime}$, extending the correspondence $f$ near the origin.

Now, we complete this section with a proof of Lemma 3.

Proof of Lemma 3. The ideas of the proof were given in [19]. Assume that $Q_{0} \subset E$. From Proposition 4.1 of [18] there exists a point $t \in \Gamma \backslash E$ such that $Q_{0} \cap Q_{t} \neq \emptyset$. Let $h: \tilde{U} \rightarrow \mathbb{C}^{n}$ be the germ of the correspondence $f$ defined in a neighborhood $\tilde{U}$ of $t$. We shrink $\tilde{U}$ and choose $V$ in such a way that for any $w \in V$, the set $Q_{w} \cap \tilde{U}$ is connected. Observe that if $V$ is small enough, then $Q_{w} \cap \tilde{U} \neq \emptyset$ for any $w \in V$, as $w \in Q_{t}$ implies $t \in Q_{w}$. Note that $V$ is a 
neighborhood of $Q_{t} \cap \tilde{U}$; since if $w \in Q_{t}$, then $t \in Q_{w}$ and $Q_{w} \cap \tilde{U} \neq \emptyset$. Let $\Sigma=\left\{z \in V: Q_{z} \cap \tilde{U} \subset S_{h}\right\}$, where $S_{h}$ is the branch locus of $h$. We define

$$
X=\left\{\left(w, w^{\prime}\right) \in(V \backslash \Sigma) \times \mathbb{C}^{n}: h\left(Q_{w} \cap \tilde{U}\right) \subset Q_{w^{\prime}}^{\prime}\right\} .
$$

As in Claim 1, $X$ extends as a complex analytic set in $(V \backslash \Sigma) \times \mathbb{C}^{n}$, and $\Sigma \times \mathbb{C}^{n}$ is a removable singularity for $X \subset V \times \mathbb{C}^{n}$. We denote also by $X$ this extension. Since $M^{\prime}$ is algebraic $X$ extends to a complex analytic set in $V \times \mathbb{P}^{n}$. We denote this variety by $\bar{X}$. Clearly, the restriction of $\bar{X}$ to $V \times\left(\mathbb{P}^{n} \backslash H_{0}\right)=V \times \mathbb{C}^{n}$ coincides with the set defined by (3.1), where $H_{0}=\left\{t_{0}=0\right\}$ is the hyperplane at infinity. Let $\pi: \bar{X} \rightarrow V$ and $\pi^{\prime}: \bar{X} \rightarrow \mathbb{P}^{n}$ be the natural projections. According to [19] (Lemma 3), $h$ extends as a holomorphic correspondence to $V \backslash\left(\Lambda_{1} \cup \Lambda_{2}\right)$, where $\Lambda_{1}=\pi\left(\pi^{\prime-1}\left(H_{0}\right)\right)$ and $\Lambda_{2}=\pi\left\{\left(w, w^{\prime}\right) \in \bar{X}: \operatorname{dim} \pi^{-1}(w) \geq\right.$ 1\}. It is easy to see that $\Lambda_{1}$ is a complex manifold of dimension at most $n-1$, and according to [18] (Proposition 3.3), $\Lambda_{2}$ is a complex-analytic set of dimension at most $n-2$. By considering dimension, we may assume that $Q_{0} \cap V \not \subset \Lambda_{2}$. Also, we may assume that $Q_{0} \cap V \not \subset \Lambda_{1}$; since otherwise we can perform a linear fractional transformation such that $H_{0}$ is mapped onto another complex hyperplane $H \subset \mathbb{P}^{n}$ with $H \cap M^{\prime}=\emptyset$. Thus, by the holomorphic extension along $Q_{t}$ we can find points in $Q_{0}$ where $h$ extends as a holomorphic correspondence. This implies that in the new coordinates $Q_{0} \not \subset E$.

\section{Extension along Segre varieties}

The propagation of analyticity of holomorphic correspondences along Segre varieties is given in the following proposition.

Proposition 1. Let $M$ be a smooth real-analytic, essentially finite hypersurface in $\mathbb{C}^{n}, n>1$, and let $U_{1} \subset \subset U_{2}$ be a standard pair of neighborhoods of a point $a \in M$. Let $f: U_{a} \rightarrow \mathbb{C}^{n}$ be a germ of a holomorphic correspondence such that $f\left(U_{a} \cap M\right) \subset M^{\prime}$, where $U_{a}$ is an arbitrary small neighborhood of $a, U_{a} \subset U_{1}$ and $M^{\prime} \subset \mathbb{C}^{n}$ is a smooth real-algebraic essentially finite hypersurface. Then there exist $V$ a neighborhood of $Q_{a} \cap U_{1}$ and an analytic set $\Lambda \subset V, \operatorname{dim}_{\mathbb{C}} \Lambda \leq n-1$ such that for any $b \in\left(Q_{a} \cap U_{1}\right) \backslash \Lambda$, the graph of $f$ extends as an analytic set to $W \times \mathbb{C}^{n}$, where $W$ is a neighborhood of the connected component of $Q_{b} \cap M \cap U_{1}$ containing a.

Proof. The proof is in two steps. First, to construct a correspondence in a neighborhood of $\left(Q_{a} \cap U_{1}\right) \backslash \Lambda$ and second to extend the graph of $f$ to an analytic set as in Proposition 1.

Step 1. We shrink $U_{a}$ and choose $V$ a neighborhood of $Q_{a} \cap U_{1}$ in such a way that for any $w \in V$, the set $Q_{w} \cap U_{a}$ is connected. Observe that if $V$ is small enough, then $Q_{w} \cap U_{a} \neq \emptyset$ for any $w \in V$, as $w \in Q_{a}$ implies $a \in Q_{w}$. Let $S_{f} \subset U_{a}$ be the branch locus of $f$ and let $\Sigma=\left\{z \in V: Q_{z} \cap U_{a} \subset S_{f}\right\}$. Since $M$ is essentially finite and $\operatorname{dim}_{\mathbb{C}} S_{f}=n-1$, by shrinking $U_{a}$ if necessary, we 
may assume that $\Sigma$ is a finite set. We define

$$
X=\left\{\left(w, w^{\prime}\right) \in(V \backslash \Sigma) \times \mathbb{C}^{n}: f\left(Q_{w} \cap U_{a}\right) \subset Q_{w^{\prime}}^{\prime}\right\} .
$$

We would like to have $Q_{w} \cap U_{a}$ connected for any $w \in V$ to avoid ambiguity in the condition $f\left(Q_{w} \cap U_{a}\right) \subset Q_{w^{\prime}}^{\prime}$; since different components of $Q_{w} \cap U_{a}$ could be mapped a priori to different Segre varieties. Similar to the proof of Claim 1, one can show that $X$ is a complex analytic set in $(V \backslash \Sigma) \times \mathbb{C}^{n}$, and that $\Sigma \times \mathbb{C}^{n}$ is a removable singularity for $X \subset V \times \mathbb{C}^{n}$. For simplicity, we denote again by $X$ the closure of $X$ in $V \times \mathbb{C}^{n}$. By the invariance property of Segre varieties (see (2.2)), $X$ extends the graph of $f_{\mid V \cap U_{a}}$ (the restriction of $f$ to $V \cap U_{a}$ ). From the algebraicity of $M^{\prime}$, the set $X$ extends to an analytic set in $V \times \mathbb{P}^{n}$. This extension will be denoted by $\bar{X}$. Let $\pi: \bar{X} \rightarrow V$ and $\pi^{\prime}: \bar{X} \rightarrow \mathbb{P}^{n}$ be the natural projections. We define the following sets : $\Lambda_{1}=\pi\left(\pi^{\prime-1}\left(H_{0}\right)\right)$ and $\Lambda_{2}=\pi\left\{\left(w, w^{\prime}\right) \in \bar{X}: \operatorname{dim} \pi^{-1}(w) \geq 1\right\}$. Here $H_{0} \subset \mathbb{P}^{n}$ is the hyperplane at infinity. Note that $\Lambda_{1}$ is a complex manifold of dimension at most $n-1$ in $\mathbb{C}^{n}$ and $\Lambda_{2}$ is a complex analytic set of dimension at most $n-2$ (see Proposition 3.3 in [18]). The projection $\pi$ is proper; since $\mathbb{P}^{n}$ is compact. Let $\Lambda=\Lambda_{1} \cup \Lambda_{2}$. Now, it is clear that the restriction $\left(\pi^{\prime} \circ \pi^{-1}\right)_{\mid V \backslash \Lambda}$ is a holomorphic correspondence extending $f_{\mid V \cap U_{a}}$.

Step 2. Consider the restriction of the extended correspondence to some neighborhood $U_{b}$ of $b, b \in\left(Q_{a} \cap U_{1}\right) \backslash \Lambda$ and $U_{b} \subset V$. Let $F: U_{b} \rightarrow \mathbb{C}^{n}$ be the corresponding multiple-valued mapping and $W$ be a neighborhood of the connected component of $Q_{b} \cap M \cap U_{1}$ that contains $a$ (we choose $W$ and we shrink $U_{b}$ so that for all $w \in W, Q_{w} \cap U_{b}$ is non-empty and connected). We denote by $S_{F}$ the branch locus of $F$ (i.e., $z \in S_{F}$ if the coordinate projection $\pi$ is not locally biholomorphic near $\left.\pi^{-1}(z)\right)$ and let $\Sigma=\left\{w \in W: Q_{w} \cap U_{b} \subset S_{F}\right\}$. Consider the set

$$
X^{*}=\left\{\left(w, w^{\prime}\right) \in(W \backslash \Sigma) \times \mathbb{C}^{n}: F\left(Q_{w} \cap U_{b}\right) \subset Q_{w^{\prime}}^{\prime}\right\} .
$$

Similar to the proof of Claim 1, one can show that $X^{*}$ is a complex analytic set in $(W \backslash \Sigma) \times \mathbb{C}^{n}$, and that $\Sigma \times \mathbb{C}^{n}$ is a removable singularity for $X^{*} \subset W \times \mathbb{C}^{n}$. We denote by $\bar{X}^{*}$ the closure of $X^{*}$ in $W \times \mathbb{C}^{n}$.

To show that $\bar{X}^{*}$ contains the graph of $f_{\mid W \cap U_{a} \cap V}$, it suffices to use the following lemma.

Lemma 5. $\bar{X}^{*} \cap\left[\left(W \cap U_{a} \cap V\right) \times \mathbb{C}^{n}\right]=X \cap\left[\left(W \cap U_{a} \cap V\right) \times \mathbb{C}^{n}\right]$.

Proof. We adapt the proof of Lemma 1 in [20] to our situation. To simplify the notations we denote $W \cap U_{a} \cap V$ by $\tilde{U}_{a}$. We need the following observation.

Claim 2. For $z \in \tilde{U}_{a}$ and $Q_{z} \cap \tilde{U}_{a} \neq \emptyset$, the following inclusions are equivalents:

(i) $f\left(Q_{z} \cap \tilde{U}_{a}\right) \subset Q_{z^{\prime}}^{\prime}$.

(ii) $F\left(Q_{z} \cap \tilde{U}_{a}\right) \subset Q_{z^{\prime}}^{\prime}$.

(iii) $F\left(Q_{z} \cap U_{b}\right) \subset Q_{z^{\prime}}^{\prime}$. 
Proof of Claim 2. (i) $\Rightarrow$ (ii) Let $w \in Q_{z} \cap \tilde{U}_{a}, w^{\prime} \in F(w)$ and $Z^{\prime} \in f(z)$. From the definition of $F$, it follows that $f\left(Q_{w} \cap \tilde{U}_{a}\right) \subset Q_{w^{\prime}}^{\prime}$. Then $z \in Q_{w}$ implies that $f(z) \subset Q_{w^{\prime}}^{\prime}$. Consequently, $w^{\prime} \in Q_{Z^{\prime}}^{\prime}$. Since $\left(w, w^{\prime}\right) \in X$ was arbitrary, it follows that $F\left(Q_{z} \cap \tilde{U}_{a}\right) \subset Q_{Z^{\prime}}^{\prime}$. From (i) and by the invariance property of Segre varieties, $Q_{Z^{\prime}}^{\prime}=Q_{z^{\prime}}^{\prime}$. Hence, $F\left(Q_{z} \cap \tilde{U}_{a}\right) \subset Q_{z^{\prime}}^{\prime}$.

(ii) $\Rightarrow$ (iii) Suppose that $F\left(Q_{z} \cap \tilde{U}_{a}\right) \subset Q_{z^{\prime}}^{\prime}$ and $Z^{\prime} \in f(z)$. Then $z^{\prime} \in I_{Z^{\prime}}^{\prime}$. Let $w \in Q_{z} \cap U_{b}$ and $w^{\prime} \in F(w)$. From the definition of $F, f\left(Q_{w} \cap \tilde{U}_{a}\right) \subset Q_{w^{\prime}}^{\prime}$. In particular, for $z \in Q_{w} \cap \tilde{U}_{a}, f(z) \subset Q_{w^{\prime}}^{\prime}$. Consequently, $w^{\prime} \in Q_{z^{\prime}}^{\prime} \cap Q_{Z^{\prime}}^{\prime}$. It follows that $F\left(Q_{z} \cap U_{b}\right) \subset Q_{Z^{\prime}}^{\prime}=Q_{z^{\prime}}^{\prime}$.

(iii) $\Rightarrow$ (i) Suppose now that $F\left(Q_{z} \cap U_{b}\right) \subset Q_{z^{\prime}}^{\prime}$. Let $w \in Q_{z} \cap U_{b}$ and $w^{\prime} \in F(w)$. Therefore, $w^{\prime} \in Q_{z^{\prime}}^{\prime}$. From the definition of $F, f\left(Q_{w} \cap U_{a}\right) \subset Q_{w^{\prime}}^{\prime}$. In particular, $f(z) \subset Q_{w^{\prime}}^{\prime}$ and then $w^{\prime} \in Q_{Z^{\prime}}^{\prime}$ for all $Z^{\prime} \in f(z)$. Consequently, $w^{\prime} \in Q_{z^{\prime}}^{\prime} \cap Q_{Z^{\prime}}^{\prime}$ for any $Z^{\prime} \in f(z)$. Since the dimension of $F\left(Q_{z} \cap U_{b}\right)$ is equal to $2 n-2$, then $z^{\prime} \in I_{Z^{\prime}}^{\prime}$ for all $Z^{\prime} \in f(z)$. This proves that $f\left(Q_{z} \cap \tilde{U}_{a}\right) \subset Q_{z^{\prime}}^{\prime}$.

Now, we continue the proof of Lemma 5. Let $\left(z, z^{\prime}\right) \in X^{*} \cap\left(\tilde{U}_{a} \times \mathbb{C}^{n}\right)$. Then $F\left(Q_{z} \cap U_{b}\right) \subset Q_{z^{\prime}}^{\prime}$. In view Claim 2, $f\left(Q_{z} \cap \tilde{U}_{a}\right) \subset Q_{z^{\prime}}^{\prime}$. This implies that $\left(z, z^{\prime}\right) \in X$. Conversely, if $\left(z, z^{\prime}\right) \in X \cap\left(\tilde{U}_{a} \cap \mathbb{C}^{n}\right)$, then $f\left(Q_{z} \cap \tilde{U}_{a}\right) \subset Q_{z^{\prime}}^{\prime}$. In view of Claim 2, $F\left(Q_{z} \cap U_{b}\right) \subset Q_{z^{\prime}}^{\prime}$. Hence, $\left(z, z^{\prime}\right) \in X^{*}$. This finishes the proof of Lemma 5 .

By using Proposition 1 and Proposition 4.1 in [18] we establish the following:

Proposition 2. Let $M \subset U \subset \mathbb{C}^{n}$ and $M^{\prime} \subset U^{\prime} \subset \mathbb{C}^{n}, n>1$, be closed hypersurfaces of finite type in some domain $U$ respectively $U^{\prime}$ in $\mathbb{C}^{n}$. We assume that $M\left(\right.$ resp. $\left.M^{\prime}\right)$ divides the domain $U$ (resp. $\left.U^{\prime}\right)$ into two connected components $U^{+}$and $U^{-}$(resp. $\left.U^{ \pm}\right)$. Assume additionally that $M$ is smooth real-analytic and $M^{\prime}$ is smooth real-algebraic. Further, suppose that $\Gamma \subset M$ is a connected open set and $\partial \Gamma \cap M$ is a smooth generic submanifold. Let $f: U^{-} \rightarrow U^{\prime}$ be a holomorphic correspondence that extends continuously up to $\Gamma$ and maps $\Gamma$ to $M^{\prime}$ and let $p$ be a point in $\partial \Gamma=\bar{\Gamma} \backslash \Gamma$. Then there exists a neighborhood $U_{p} \ni p$ in $\mathbb{C}^{n}$ such that the graph of $f$ extends as an analytic set to $U_{p} \times \mathbb{C}^{n}$.

Proof. According to [23], $f$ extends as a holomorphic correspondence in a neighborhood of $\Gamma$. Without loss of generality we may assume that $f$ is not constant. Let $U_{1} \subset \subset U_{2}$ be a standard pair of neighborhoods of $p$. According to [18], there exists an open subset $\omega \subset Q_{p} \cap U_{1}$ such that for all $b \in \omega, Q_{b} \cap \Gamma \neq \emptyset$. Moreover, there exists a non-constant closed path $\gamma \subset\left(Q_{b} \cap \Gamma\right) \cup\{p\}$ with the end point at $p$. In view of [18] (Remark 2 following Proposition 4.1) $Q_{b}$ intersects $\Gamma$ transversally. Let $\Lambda=\Lambda_{1} \cup \Lambda_{2}$ be the set defined in Proposition 3.1. Recall that $\operatorname{dim}_{\mathbb{C}} \Lambda_{2} \leq n-2$ and $\operatorname{dim}_{\mathbb{C}} \Lambda_{1} \leq n-1$. We may choose the triplet $(b, \gamma, a)$ such that $b \in \omega \backslash \Lambda_{2}$ and $a \in \gamma \subset Q_{b} \cap \Gamma$. Notice that $\operatorname{dim}_{\mathbb{C}}\left(Q_{a} \cap \omega\right)=n-2$ and $b \in Q_{a} \cap \omega$. Since $b \in Q_{a} \cap \omega$, then $Q_{a} \cap \omega \not \subset \Lambda_{2}$. Also, we may exclude the case when $Q_{a} \cap \omega \subset \Lambda_{1}$. Indeed; if $Q_{a} \cap \omega \subset \Lambda_{1}$, we can perform a linear-fractional transformation in $\mathbb{P}^{n}$ such $H_{0}$ is mapped 
onto another complex hyperplane $H \subset \mathbb{P}^{n}$ with $H \cap M^{\prime}=\emptyset$. Hence, we may assume that $\left(Q_{a} \cap \omega\right) \not \subset \Lambda$ and if necessary, we may replace $b$ by another point in $\left(Q_{a} \cap \omega\right) \backslash \Lambda$. Now, the result follows from Proposition 1.

\section{Proof of Theorem 1}

Let $M_{\mathrm{h}}$ be the set of points $z \in M$, where $f$ extends as a holomorphic correspondence to a neighborhood of $z$. According to [23], $M_{\mathrm{h}}$ is not empty. It is open by construction. To prove Theorem 1, it suffices to show that $M_{\mathrm{h}}$ is closed in $M$. We argue by contradiction. Assume that $\bar{M}_{\mathrm{h}} \neq M_{\mathrm{h}}$ and let $q \in \partial M_{\mathrm{h}}=\bar{M}_{\mathrm{h}} \backslash M_{\mathrm{h}}$. We follow the idea in [22], used also in [1]. Since $M$ is globally minimal, there exists a CR-curve $\gamma$ (i.e., the tangent vector to $\gamma$ at any point is contained in the complex tangent to $M$ ) passing through $q$ and entering $M_{\mathrm{h}}$. We will use the construction of a family of ellipsoids used by Merker and Porten [14].

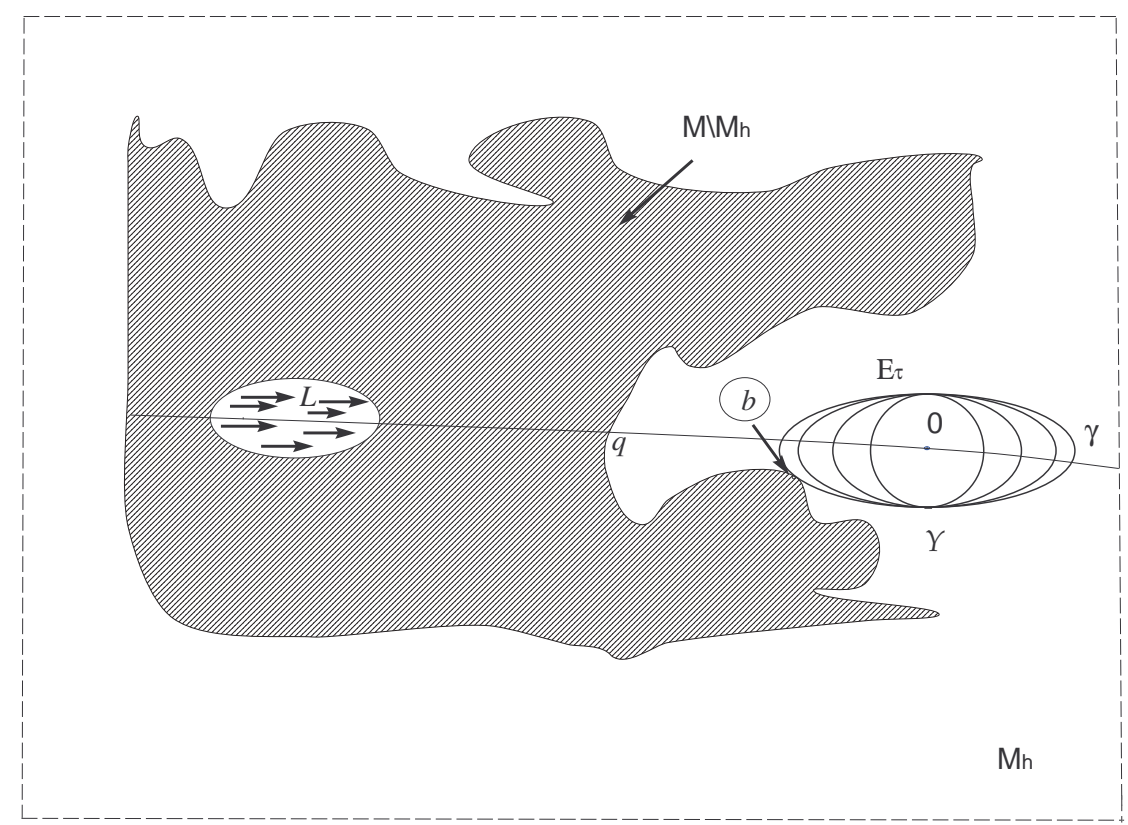

Figure 1. Construction of ellipsoids

After shortening $\gamma$ we may assume that $\gamma$ is a smoothly embedded segment. Then $\gamma$ can be described as a part of an integral curve of some non-vanishing smooth CR vector field $L$ (i.e., section of $T^{c} M$ ) near $q$. Integrating $L$, we obtain a smooth coordinate system $(t, s) \in \mathbb{R} \times \mathbb{R}^{2 n-2}$ on $M$ such that for any fixed $s_{0}$ the segments $\left(t, s_{0}\right)$ are contained in the trajectories of $L$. After a 
translation, we may assume that $0 \in \gamma \cap M_{\mathrm{h}}$ and 0 is close to $q$. For $\epsilon>0$ and $\tau>0$ we define the family of ellipsoids on $M$ centered at 0 by

$$
E_{\tau}=\left\{(t, s):|t|^{2} / \tau+|s|^{2}<\epsilon\right\} .
$$

Observe that every $\partial E_{\tau}$ is transverse to the trajectories of $L$ out off the set $\Upsilon=\left\{(0, s):|s|^{2}=\epsilon\right\}$. So, $\partial E_{\tau}$ is generic at every point except the set $\Upsilon$. Note that $\Upsilon$ is contained in $M_{\mathrm{h}}$. Fix $\epsilon>0$ so small such that for some $\tau_{0}>0$ the ellipsoid $E_{\tau_{0}}$ is compactly contained in $M_{\mathrm{h}}$. Let $\tau_{1}$ be the smallest positive number such that $f$ does not extend holomorphically to some point $b \in \partial E_{\tau_{1}}$. Note that $\tau_{1}>\tau_{0}$ and $b$ may be different from $q$. The contradiction is to show that $f$ extends as a holomorphic correspondence to a neighborhood of $b$. Near $b, \partial E_{\tau_{1}}$ is a smooth generic manifold of $M$; since the non-generic points of $\partial E_{\tau_{1}}$ are contained in $\Upsilon$, which is contained in $M_{\mathrm{h}}$. By Proposition 2, there exists a neighborhood $U_{b} \ni b$ in $\mathbb{C}^{n}$ such that the graph of $f$ extends as an analytic set to $U_{b} \times \mathbb{C}^{n}$. In view of Theorem $2, f$ extends holomorphically to a neighborhood of $b$. This contradiction proves that $M_{\mathrm{h}}=M$ and finishes the proof of Theorem 1 .

\section{References}

[1] B. Ayed and N. Ourimi, Analytic continuation of holomorphic mappings, C. R. Math. Acad. Sci. Paris 347 (2009), no. 17-18, 1011-1016.

[2] __ A Local extension of proper holomorphic maps between some unbounded domains in $\mathbb{C}^{n}$, Bull. Belg. Math. Soc. Simon Stevin 17 (2010), no. 3, 513-534.

[3] M. S. Baouendi and L. P. Rotshschild, Germs of CR maps between real analytic hypersurfaces, Invent. Math. 93 (1998), no. 3, 481-500.

[4] __ Images of real hypersurfaces under holomorphic mappings, J. Differential Geom. 36 (1992), no. 1, 75-88.

[5] F. Berteloot and A. Sukhov, On the continuous extension of holomorphic correspondences, Ann. Scuola Norm. Sup. Pisa Cl. Sci. (4) 24 (1997), no. 4, 747-766.

[6] E. M. Chirka, Complex Analytic Sets, Kluwer Academic Publishers, 1989.

[7] S. Chern and J. Moser, Real hypersurfaces in complex manifolds, Acta Math. 133 (1974), $219-271$.

[8] K. Diederich and J. E. Fornaess, Proper holomorphic mappings between real-analytic pseudoconvex domains in $\mathbb{C}^{n}$, Math. Ann. 282 (1988), no. 4, 681-700.

[9] K. Diederich and S. Pinchuk, Analytic sets extending the graphs of holomorphic mappings, J. Geom. Anal. 14 (2004), no. 2, 231-239.

[10] , Proper holomorphic maps in dimension 2 extend, Indiana Univ. Math. J. 44 (1995), no. 4, 1089-1126.

[11] _ Regularity of continuous CR maps in arbitrary dimension, Mich. Math. J. 51 (2003), no. 1, 111-140.

[12] K. Diederich and J. Fornaess, Proper holomphic mapping between real-analytic pseudoconvex domains in $\mathbb{C}^{n}$, Math. Ann. 282 (1988), no. 4, 681-700.

[13] K. Diederich and S. Webster, A reflection principle for degenerate real hypersurfaces, Duke Math. J. 47 (1980), no. 4, 835-845.

[14] J. Merker and E. Porten, On wedge extendability of CR-meromorphic functions, Math. Z. 241 (2002), no. 3, 485-512.

[15] S. Pinchuk, On holomorphic maps of real-analytic hypersurfaces, Math. USSR Sb. 34 (1978), 503-519. 
[16] Analytic continuation of holomorphic mappings and the problem of holomorphic classification of multidimensional domains, Doctoral dissertation (habilitation), Moscow State Univ., 1980.

[17] S. Pinchuk and K. Verma, Analytic sets and the boundary regularity of CR mappings, Proc. Amer. Math. Soc. 129 (2001), no. 9, 2623-2632.

[18] R. Shafikov, Analytic continuation of germs of holomorphic mappings, Mich. Math. J. 47 (2000), no. 1, 133-149.

[19] _ Analytic continuation of holomorphic correspondences and equivalence of domains in $\mathbb{C}^{n}$, Invent. Math. 152 (2003), no. 3, 665-682.

[20] _ On boundary regularity of proper holomorphic mappings, Math. Z. 242 (2002), no. 3, 517-528

[21] R. Shafikov and K. Verma, A local extension theorem for proper holomorphic mappings in $\mathbb{C}^{2}$, J. Geom. Anal. 13 (2003), no. 4, 697-714.

[22] _ Extension of holomorphic maps between real hypersurfaces of different dimension, Ann. Inst. Fourier, Grenoble 57 (2007), no. 6, 2063-2080.

[23] B Boundary regularity of correspondences in $\mathbb{C}^{n}$, Proc. Indian Acad. Sci. (Math. Sci.) 116 (2006), no. 1, 59-70.

[24] K. Verma, Boundary regularity of correspondences in $\mathbb{C}^{2}$, Math. Z. 231 (1999), no. 2, $253-299$.

[25] S. M. Webster, On the mapping problem for algebraic real hypersurfaces, Invent. Math 43 (1977), no. 1, 53-68.

Department of Mathematics

King SAud University

P. O. Box 2455, Riyadh 11451 King SAudi Arabia

Current Address:

Faculté des Sciences de Bizerte

JARZOUNA, 7021, TUNISIA

E-mail address: ourimin@yahoo.com 